\title{
Five novel TTR variants: associated phenotypes and structural consequences
}

\author{
Dorota Rowczenio*, Helen Lachmann, Ashutosh Wechalekar, Janet Gilbertson, Marianna Fontana, Carol Whelan, \\ Julian Gillmore, Philip Hawkins \\ From First European Congress on Hereditary ATTR amyloidosis \\ Paris, France. 2-3 November 2015
}

\section{Objective}

Transthyretin (ATTR) amyloidosis is the commonest form of hereditary amyloidosis. More than 120 amyloidogenic TTR mutations have been described and with few exceptions, these are rare, reported only in isolated kindreds. We describe here the clinical characteristics and disease course in 11 patients with novel TTR mutations. The structural consequence of replacing the wildtype amino acid with variant was examined in silico using PyMOL, an online molecular visualization tool.

\section{Methods}

11 individuals with features raising the possibility of ATTR amyloidosis were referred to the UK NAC for clinical and laboratory investigation.

\section{Results}

Median (range) age at onset of symptoms was 58 (44-68) years; median age at diagnosis was 61 (46-74) years. Three patients presented with progressively worsening shortness of breath and chest tightness on exertion, six suffered with gradually progressive neuropathy, one was hospitalised with heart failure and oedema whilst one was asymptomatic but had a family history of FAP. Amyloid deposits were identified in biopsies in all cases by Congo red staining and were confirmed to be of ATTR type by immunohistochemistry. Genetic analysis revealed five novel variants: p.E74L (E54L), p.E74Q (E54Q), p.A101V (A81V), p.H110D (H90D) and p.I127F (I107F). The cohort was followed for a median (range) of 4 (1-7) years after referral during which six patients died of cardiac amyloidosis. Median (range) age at death was 66 (60-80) years, and median (range) time from onset of symptoms to death was 8 (4-16) years.

\footnotetext{
National Amyloidosis Centre, University College London, NW3 2PF, London,
} UK
Cite this article as: Rowczenio et al:: Five novel TTR variants: associated phenotypes and structural consequences. Orphanet Journal of Rare Diseases 2015 10(Suppl 1):P28. 